\title{
Auf die Plätze, fertig - Mahlzeit! Praxis der Sporternährung für verschiedene Belastungsbereiche: Raumfahrt
}

\author{
Ready, Steady - Enjoy Your Meal! \\ The Practice of Sports Nutrition at Different Intensity Levels: Space Travel
}

Autor

Institut

\section{Abeln}

Institut für Bewegungs- und Neurowissenschaft, Deutsche Sporthochschule Köln
Schlüsselwörter

- Ernährungsplanung

- Appetit

- Sport

- Kalorienverbrauch

- Nährstoffbedarf

- degenerative Prozesse

- Befinden

Keywords

nutrition planning

- appetite

- exercise/sports

- calorie consumption

- nutrient requirement

- degenerative processes

- wellbeing

\section{Zusammenfassung \\ $\nabla$}

Bei Weltraummissionen ist eine ausreichende und adäquate Ernährung die Grundvoraussetzung für die Gesundheit der Astronauten. Die Ernährungsplanung ist jedoch mit vielen Unsicherheiten behaftet und basiert größtenteils auf Studien, die auf der Erde durchgeführt und auf die Bedingungen im Weltraum übertragen werden. Sport wirkt sich positiv sowohl auf die körperliche Leistungsfähigkeit als auch auf das Befinden aus. Aus diesem Grund trainieren Astronauten in der Regel 2,5 Stunden pro Tag. Sport wird bei der Ernährungsplanung bisher jedoch unzureichend berücksichtigt. Das birgt die Gefahr einer Unterversorgung, die Abbauprozesse im All begünstigt. Wie der Ernährungsplan an dieses Sportprogramm angepasst werden muss, ist fraglich. Weder der sportbedingte Kalorienverbrauch noch der Nährstoffbedarf im Weltraum sind derzeit ausreichend geklärt. Grundsätzlich dienen wissenschaftliche Studien auf der Erde der Ernährungsplanung im Weltall und umgekehrt.

\section{Abstract \\ $\nabla$}

During space missions, sufficient and adequate nutrition is a fundamental requirement for the health of the astronauts. Nutrition planning is, however, be set by many insecurities and uncertainties and is mostly based on studies that have been conducted on Earth and are transferred to conditions in space. Exercise has a positive effect on physical performance ability as well as mood. For this reason, astronauts usually exercise for two and a half hours per day. However, such exercise is insufficiently considered during nutrition planning. This entails the risk of underprovision, which encourages degenerative processes in space. Questions remain over how nutrition plans should be adapted to this exercise programme. Neither exercise-related calorie requirements nor nutrient requirements in space are currently sufficiently well known. Basically, scientific studies in Earth are used to plan nutrition in space and vice versa.

\section{Bibliografie}

Dol http://dx.doi.org/ 10.1055/s-0042-102740 Aktuel Ernahrungsmed 2016; 41, Supplement 1: S29-S31 (c) Georg Thieme Verlag KG Stuttgart · New York ISSN 1862-0736

\section{Korrespondenzadresse}

\section{Dr. Vera Abeln}

Institut für Bewegungs- und Neurowissenschaft, Deutsche Sporthochschule Köln Am Sportpark Müngersdorf 6 50933 Köln

Tel.: 0221/4982-4210

v.abeln@dshs-koeln.de
Zu Beginn der Weltraumfahrt Anfang der 1960erJahre bestand die Nahrung für die Astronauten aus Tuben, Pasten und Tabletten. Das war für die damals sehr kurzen Missionen von mehreren Stunden bis zu einem Tag vertretbar. Heute ähnelt die Astronautenkost einer Kantinenmahlzeit mit echten Lebensmitteln - eine wichtige Anpassung angesichts zunehmender Missionsdauer und der Planung von Weltraumaufenthalten von Monaten bis Jahren (z.B. Marsexploration).

Die Ernährungsplanung für Weltraummissionen ist eine komplexe Herausforderung. Astronauten sind physisch und psychisch mit Bedingungen konfrontiert, die sich auf die Nahrungsqualität und den Appetit auswirken: Abgesehen davon, dass das Essen im Spaceshuttle weniger frisch und viel eintöniger ist als auf der Erde, entwickeln viele Astronauten eine Bewegungskrankheit (engl. Motion sickness), die mit Übelkeit einhergeht. Die schwerelosigkeitsbedingte Verschiebung der Körperflüssigkeiten in Richtung obere Körperhälfte verursacht ebenso wie die sehr trockene Luft häufig Verstopfung. Die Atmosphäre im Weltall, vor allem Temperaturunterschiede, veränderter Luftdruck, Luftkomposition und höhere Strahlung, beeinträchtigt den Geruch und Geschmack. Darüber hinaus wirken sich das veränderte Aktivitätsprofil und psychosoziale Stressfaktoren auf das Essverhalten aus. Die Astronauten sind zum Teil über Monate im Spaceshuttle isoliert und eingesperrt, weit entfernt von zu Hause, Familie und Freunden. Daher kommt dem Essen, neben der grundsätzlichen Ernährung, eine große psychische Bedeutung zu. Ein Stück 


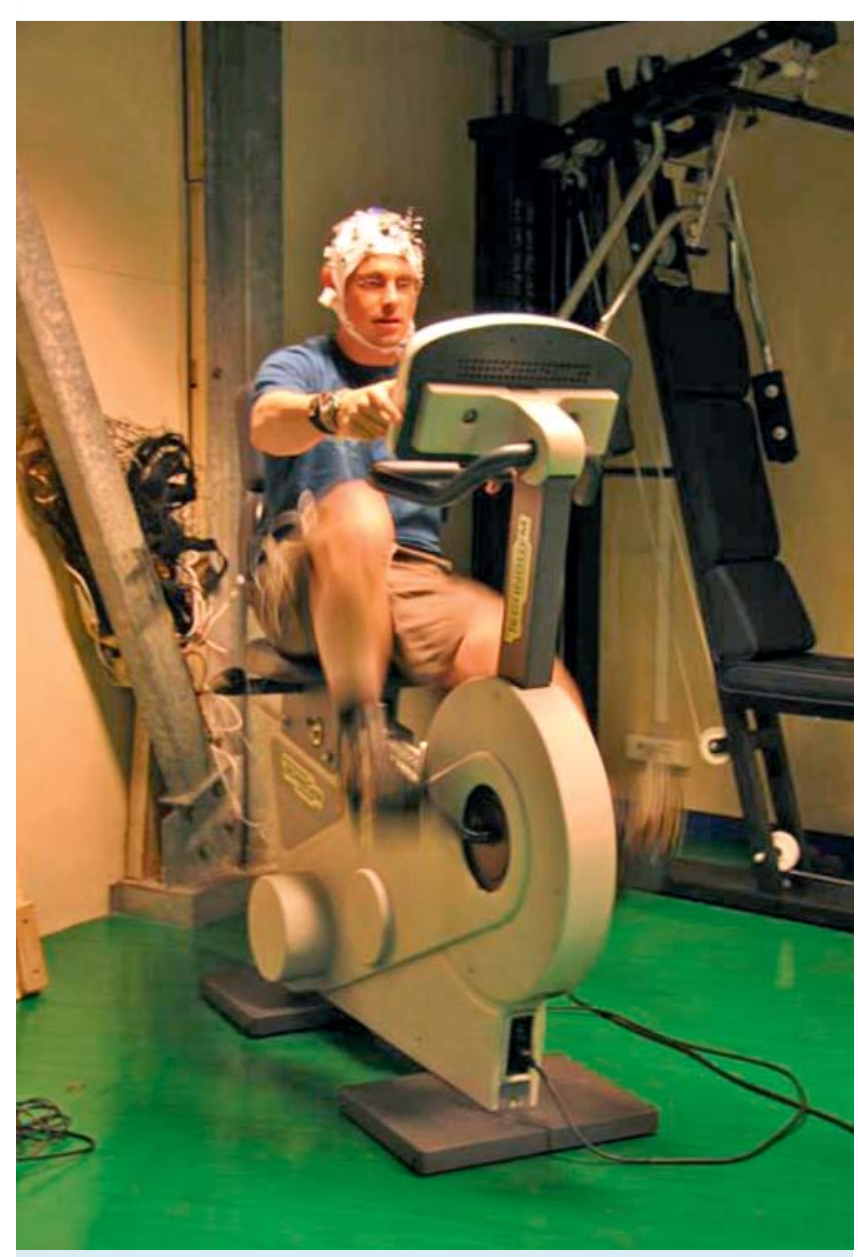

Abb. 1 Proband während eines Isolations-Projektes in der Antarktis auf der Concordia Forschungsstation bei der sportlichen Betätigung auf dem Fahrradergometer. Copyright: "Eoin MacDonald-Nethercott, ESA medicaldoctor on site".

Schokolade kann in dieser Situation ein wichtiger Seelentröster sein.

Grundlegend stellt sich die Frage: Wie viele und welche Lebensmittel können angesichts der begrenzten Kapazität mit an Bord? Zudem sollte bei der Ernährungsplanung berücksichtigt werden, dass sich der menschliche Körper während des Aufenthalts im Weltall an die neuen Bedingungen adaptiert und in seinen Ansprüchen an die Ernährung nicht mehr dem auf der Erde gleicht.

\section{Worauf basiert die Ernährungsplanung?}

$\nabla$

Die Ernährungsplanung für Astronauten basiert zum Teil auf Spekulation, einer spärlichen Datenlage der Weltraumforschung und zu weiten Teilen auf irdischen Studien [1]. Darüber hinaus greifen Wissenschaftler auch auf die allgemeinen Empfehlungen der WHO [2, 3] zurück.

In der Schwerelosigkeit kommt es zu gravierenden degenerativen Prozessen, etwa zum Abbau der kardiovaskulären Leistungsfähigkeit, der Muskulatur, der Knochen und Knorpel [4-6]. Da Sport diesen Prozessen entgegenwirken kann, ist regelmäßiges Training für Astronauten unerlässlich [7-9]. Zwar ist es bislang nicht gelungen, ein Sportprogramm zu entwickeln, das alle degenerativen Prozesse aufhält [10], aber sie werden laufend optimiert. Dass davon nicht nur die Physis, sondern auch die Psyche profitiert, belegen unter anderem Daten aus dem Mars 500-Projekt - eine simulierte Reise zum Mars, bei der sechs Probanden 520 Tage in einem nachgebauten Spaceshuttle in Moskau verbrachten [11]. Die Befindlichkeit der Probanden war nach absolvierten Sporteinheiten deutlich besser als vorher. Eine ähnliche Studie wurde in der Antarktis durchgeführt: Probanden, die sich sportlich betätigten, konnten ihr Befinden über 10 Monate Isolation stabil halten, während die Befindlichkeit inaktiver Probanden deutlich schlechter war ( $\bullet$ Abb. 1) [12].

\section{Sportgeräte im Weltraum}

$\nabla$

Die drei Haupttrainingsgeräte im Weltraum sind:

- ARED (Advanced Resistive Exercise Device): ein multifunktionales Krafttrainingsgerät, das für verschiedene Übungen umgebaut werden kann.

- T2 COLBERT Treadmill: ein Laufband, das die Astronauten über ein an der Hüfte befestigtes Seilzugsystem am Boden hält.

- CEVIS: ein Fahrradergometer.

Ein typischer Trainingsplan für Astronauten sieht jeden Tag 2,5 Stunden Sport vor - 90 min Krafttraining am ARED plus 60 min Ausdauertraining auf dem Laufband und Fahrradergometer im Wechsel. Diese 2,5 Stunden Sport pro Tag reichen jedoch nicht aus, um degenerative Prozesse aufzuhalten.

In der Ernährungsplanung werden derzeit nur 120 statt 150 min Sport berücksichtigt. Dabei gehen die Wissenschaftler von der Situation auf der Erde aus und übertragen sie 1:1 auf die Situation im Weltraum. Die Kernfrage ist jedoch, ob der Kalorienverbrauch beim Sport auf der Erde tatsächlich dem im Weltraum entspricht. Einige Studien sprechen dafür [3], andere haben einen erhöhten Kalorienverbrauch ermittelt [13]. Unterm Strich liegen bislang keine Daten vor, mit denen der sportbedingte Kalorienverbrauch von Astronauten kalkuliert werden kann, um den Ernährungsplan entsprechend anzupassen. Dazu kommt, dass akuter Stress durch endokrine Reaktionen die Vorausberechnungen und potenziell wirksame Gegenmaßnahmen beeinträchtigen kann [14]. Offene Punkte betreffen nicht nur den Energieverbrauch, sondern auch die Energiezufuhr: Studien ergaben, dass die Energiezufuhr im Weltraum im Vergleich zur Erde nur 70-80\% beträgt [1]. Diese Unterversorgung begünstigt Abbauprozesse. Entsprechend haben Astronauten im Schnitt 5-10\% Gewichtsverlust, der nicht nur Fett-, sondern auch Muskelmasse betrifft.

Der Nährstoffbedarf im Weltraum ist ebenfalls ein Bereich mit vielen Fragezeichen. Einige Nährstoffe werden im Weltraum bewusst erhöht dosiert, um Abbauprozessen vorzubeugen. Dazu gehören Vitamin K, Vitamin $B_{12}$ und Calcium [15]. Neben Erkenntnissen aus Ernährungsstudien auf der Erde und Weltraumstudien tastet man sich hier Schritt für Schritt auf der Suche nach dem adäquaten Nährstoffbedarf im Weltall vor. Das Ziel ist hier sicherlich noch nicht erreicht.

\section{Synergieeffekte durch Sport und optimale Ernährung}

$\nabla$

Derzeit untersuchen mehrere Forschungsarbeiten Zusammenhänge zwischen Ernährung und Sport im Weltraum. Untersucht wird zum Beispiel, wie sich Sport in Verbindung mit Natrium oder Omega-3-Fettsäuren positiv auf die Knochen auswirken oder welche Rolle das Verhältnis von Protein zu Kalium spielt [16]. Omega-3-Fettsäuren werden daneben zum Beispiel als Schutzfaktor gegen Muskelabbau oder gegen das Krebsrisiko auf- 
grund der erhöhten Strahlung diskutiert. Des Weiteren zeigte eine Gruppe erstmals, dass die doppelte Gabe von Vitamin D in Verbindung mit einer adäquaten Energieaufnahme und Sport zum Erhalt der Knochenfestigkeit im Bereich der Hüft- und Oberschenkel geführt hat [7]. Weitere Untersuchungen sind jedoch notwendig, um die optimale Balance zwischen Nährstoffzufuhr und -verbrauch insbesondere im Zusammenhang mit Sport zu finden [17]

\section{Win-Win-Situation}

$\nabla$

Die Weltraumforschung profitiert von Studien auf der Erde, aber umgekehrt profitieren Wissenschaftler auch von der Weltraumforschung, weil diese meistens unter kontrollierten Bedingungen stattfindet und weil viele degenerative Prozesse im Weltraum aufgrund der Schwerelosigkeit im Zeitraffer stattfinden. So gelang es im Rahmen des Isolationsprojektes Mars-500 zur Vorbereitung von Weltraumfahrten erstmals unter kontrollierten Bedingungen zu zeigen, dass die Salzzufuhr im Essen direkten Einfluss auf den Blutdruck hat: Parallel zur Reduktion der Salzzufuhr von 12 auf $6 \mathrm{~g}$ sank auch der systolische Blutdruck [18].

\section{Interessenkonflikt}

$\nabla$

Die Autorin hat keinen Interessenkonflikt.

\section{Literatur}

1 Smith SM, Rice BL, Dlouhy $H$ et al. Assessment of nutritional intake duing space flight and space flight analogs. Procedia Food Science 2013; $2: 27-34$

2 WHO. CINDI dietary guide. http://www.euro.who.int/_data/assets/ pdf_file/0010/119926/E70041.pdf2000 [cited 2015 23. November]

3 Lane HW, Gretebeck RJ, Schoeller DA et al. Comparison of ground-based and space flight energy expenditure and water turnover in middleaged healthy male US astronauts. Am J Clin Nutr 1997; 65: 4-12 PubMed PMID: 8988906

4 Aubert AE, Beckers F, Verheyden B. Cardiovascular function and basics of physiology in microgravity. Acta Cardiol 2005; 60: 129-151

5 Clement G, Bukley A. Artificial Gravity. Berlin: Springer; 2007
6 Bailey JF, Hargens AR, Cheng KK et al. Effect of microgravity on the biomechanical properties of lumbar and caudal intervertebral discs in mice. J Biomech 2014; 47: 2983-2988; Doi: 10.1016/j.jbiomech. 2014.07.005 PubMed PMID: 25085756

7 Smith SM, Heer MA, Shackelford LC et al. Benefits for bone from resistance exercise and nutrition in long-duration spaceflight: Evidence from biochemistry and densitometry. J Bone Miner Res 2012; 27: 1896-1906; Doi: 10.1002/jbmr.1647 PubMed PMID: 22549960

8 Ploutz-Snyder LL, Downs M, Ryder J et al. Integrated resistance and aerobic exercise protects fitness during bed rest. Med Sci Sports Exerc 2014; 46: 358-368; Doi: 10.1249/MSS.0b013e3182a62f85 PubMed PMID: 24441215

9 Fraser KS, Greaves DK, Shoemaker JK et al. Heart rate and daily physical activity with long-duration habitation of the International Space Station. Aviat Space Environ Med 2012; 83: 577-584; PubMed PMID: 22764612

10 Gopalakrishnan R, Genc KO, Rice AJ et al. Muscle volume, strength, endurance, and exercise loads during 6-month missions in space. Aviat Space Environ Med 2010; 81: 91 - 102; PubMed PMID: 20131648

11 Schneider S, Brümmer V, Carnahan $H$ et al. Exercise as a countermeasure to psycho-physiological deconditioning during long-term confinement. Behavioural Brain Research 2010; 211: 208-214; Epub 2010/03/30 Doi: 10.1016/j.bbr.2010.03.034 PubMed PMID: 20346985

12 Abeln V, MacDonald-Nethercott E, Piacentini MF et al. Exercise in isolation - a countermeasure for electrocortical, mental and cognitive impairments. Plos One 2015; 10: e0126356; Doi: 10.1371/journal.pone. 0126356 PubMed PMID: 25961821; PubMed Central PMCID: PMCPMC4427298

13 Kasyan IL, Makarov GF. External respiration, gas exchange and energy expenditure of man in weightlessness. Kosm Biol Aviakosm Med 1984; 18: 4-9

14 Lane HW, Gretebeck RJ, Smith SM. Nutrition, endocrinology, and body composition during space flight. Nutr Res 1998; 18: 1923 -1934; PubMed PMID: 11541547

15 NASA. NASA Space Flight Human-System Standard. Washington: National Aeronautics and Space Administration; 2015

16 Zwart SR, Pierson D, Mehta $S$ et al. Capacity of omega-3 fatty acids or eicosapentaenoic acid to counteract weightlessness-induced bone loss by inhibiting NF-kappaB activation: from cells to bed rest to astronauts. J Bone Miner Res 2010; 25: 1049-1057; PubMed PMID: 19874203 Doi: $10.1359 / j b m r .091041$

17 Stein TP. The relationship between dietary intake, exercise, energy balance and the space craft environment. Pflugers Arch 2000; 441 (23 Suppl): R21 - 31; PubMed PMID: 11200976

18 Rakova N, Juttner K, Dahlmann A et al. Long-term space flight simulation reveals infradian rhythmicity in human $\mathrm{Na}(+)$ balance. Cell Metab 2013; 17: 125 -131. doi: 10.1016/j.cmet.2012.11.013. PubMed PMID: 23312287 PubMed PMID: 23312287 Doi: 10.1016/j.cmet.2012.11.013 